Case Report

\title{
An Unusual Presentation of Lung Cancer
}

\author{
Likith Rai ${ }^{1}$, Amol Dilip Amonkar ${ }^{2}$ \\ ${ }^{1}$ Assitant Professor, ${ }^{2}$ Post graduate student, Department of Surgery, K.S. Hegde M edical Academy, Deralakatte, M angalore. \\ *Corresponding Author : Amol Amonkar, Department of General Surgery, K.S. Hegde M edical Academy, Mangalore, \\ Mobile : +919886535829 E-mail : amonkaramol@gmail.com
}

Received

: 06.11.2015

Review Completed : 09.11.2016

Accepted

: 01.01.2017

Keywords : Soft tissue metastasis, lung cancer, anterior abdominal wall,

\begin{tabular}{|c|}
\hline Access this article online \\
\hline Quick Response Code \\
\hline
\end{tabular}

\begin{abstract}
Soft-tissue metastasis refers to the growth of cancer cells originating from internal cancer in the soft tissues. In most cases, soft-tissue metastasis develops after initial diagnosis of the primary internal malignancy and late in the course of the disease. In very rare cases, they may occur at the same time or before the primary cancer has been detected. In our case, the soft-tissue metastasis and the primary lung cancer were diagnosed at the same time. To the best of our knowledge soft tissue metastasis on the anterior abdominal wall of unknown primary lung cancer is an extremely rare condition and only a handful of cases have been reported till date We report a case of unsuspected soft tissue metastasis on the anterior abdominal wall of an unknown primary lung cancer with relevant discussion on the same.
\end{abstract}

\section{Introduction}

Distant metastasis to soft tissue (ST) defined as metastasis to skeletal muscle and subcutaneous tissues, are rarely reported in the literature[1].Autopsy series have reported ST metastasis in $0.75-9 \%$ of patients who died of metastatic carcinoma[2].ST metastasis from lung cancer are uncommon with a reported overall prevalence of 2.3\%[3].The detection of ST metastasis may affect staging and prognosis [2].We report a 70 year old male who presented with a painless swelling over the anterior abdominal wall as the initial presentation of the unknown primary lung cancer.

\section{Case Report}

We report a 70 year old male who presented a painless swelling over the anterior abdominal wall since 20 days. He was a chronic smoker with 45 year pack history. He also noted an unintentional $10 \mathrm{~kg}$ weight loss in the past 2 months On physical examination, he had decreased muscle mass and looked cachectic, A $3 \times 3 \mathrm{cms}$ single oval swelling in the left hypochondrium in the left midclavicular line, which was firm ,mobile and become more prominent on head raising test suggestive of swelling arising from the abdominal wall ,skin over the swelling was normal .No other lesion noted anywhere else in the body.Ultrasound guided FNAC revealed metastatic poorly differentiated carcinoma. Chest $\mathrm{x}$-ray revealed a well-defined opacity in the right lower lung zone continuous with the descending pulmonary artery. CECT-thorax showed a right lower lobe lung mass with hiliar and mediastinal nodes.Bronchoscopy showed occlusion of medial and anterior basal segmental openings .Biopsy revealed poorly differentiated squamous cell carcinoma .CECT abdomen showed a well- defined lesion in the left rectus muscle suggestive of a soft tissue metastasis on the anterior abdominal wall .The patient received a palliative radiotherapy for 10 days and expired within a month of the final diagnosis.

\section{Discussion}

Lung cancer is the leading cause of cancer-related deaths [1].Approximately $50 \%$ of cases are metastatic at the time of diagnosis and $60 \%$ of patients have microscopic or 


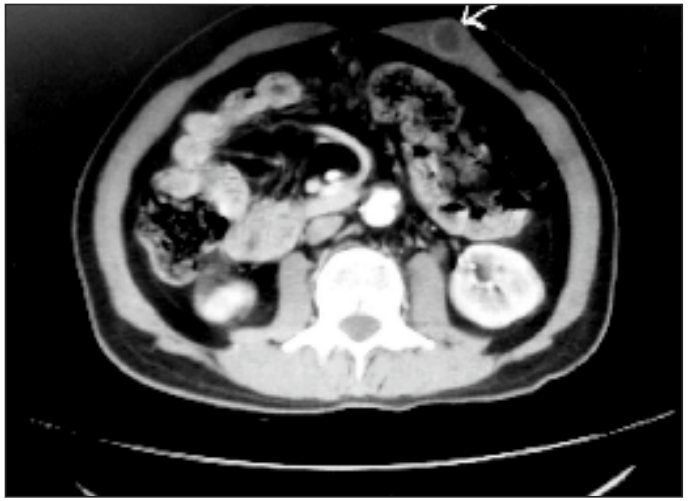

Fig. 1 : CECT abdomen showed well defined lesion in right rectus muscle suggestive of a soft tissue metastasis in the anterior abdominal wall.

clinically evident metastasis at the time of primary tumour treatment[1].Lung cancer can metastasize to any organ ,major sites include the liver (33-40\%), adrenal glands(18$38 \%)$, brain (15-43\%), bone (19-33\%), kidney (16-43\%) and abdominal lymph nodes (29\%)[1].A prospective study by salvatierra et al [4] found that patients with adenocarcinoma or large cell carcinoma were at a significantly higher risk for extra thoracic metastasis compared with patients with squamous cell carcinoma. ST metastasis are perceived as a sign of advanced disease and are regarded as a grave prognostic indicator[1].M etastasis from lung cancer are microscopically indistinguishable from metastasis of other cancers .Usually they present as fast growing solitary or multiple nodules with diameter of $5 \mathrm{~mm}-10 \mathrm{cms}$ and are firm, mobile and are covered with normal skin.[1] Sometimes, exudative or ulcerative lesions are also seen[5].They usually present as painless nodules[6].The most frequently reported locations for ST

\section{References}

1. Baldeo C,Ali R,Sreeram V et al .Lung cancer presenting as a soft tissue metastasis. Case Reponcol.2015 Jan-Aprj 8(1) : 183-188

2. Nguyen NC, Chaar BT, Osman MM Prevalence and patterns of soft tissue metastasis: detection with true wall body F-18 FDG PET/CT. BM C Med Imaging. 2007; 7:8

3. Sinha N, Niazi M, Diaz-Fuentes U, Duncalf R. An innocent appearing subcutaneous nodules diagnosis a small cell lung cancer in a never smoker female. Case Rep Onco M ed 2014; 2014:268404

4. Salvatierra A, Baamonde C, Llamas JM, Cruz F, Lopez- Pujol J. Extra thoracic staging of bronchogenic carcinoma. Chest.1990; 97: 10531058

5. Pathak S, Joshi SR, Jaison J, Kendre D. Cutaneous metastasis from carcinoma of lung. Indian Dermatol Online J.2013; 4 : 185-187

6. Hu S-S, Chen G-S, Lu Y-W, Wu C-S, Lan C-C. Cutaneous metastasis from different internal malignancies; a clinical and prognostic appraisal. J Eur Acad Dermatol Venereol. 2008; 22: 735-740.

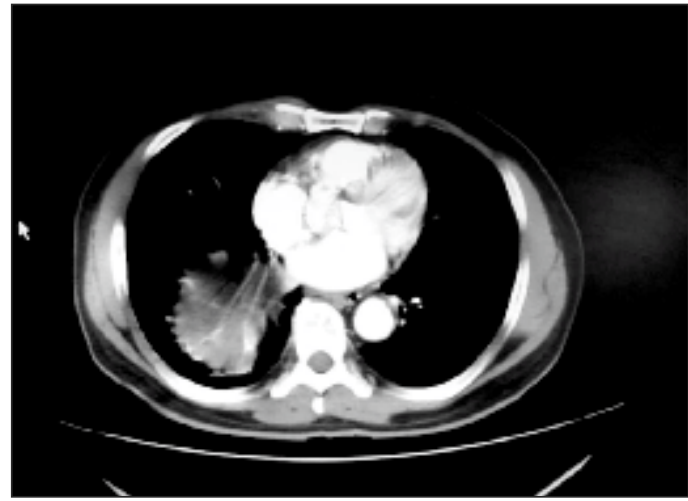

Fig. 2 : CECT Thorax showed right lower lobe lung mass with hilar and mediastinal nodes.

metastasis have been back, chest wall and abdomen[2].Rapid growth of skin nodules is an alarming sign of metastasis .Additionally, the significant weight loss in our patient indicated a possibility of malignancy, together with the history of being a chronic smoker ,timely biopsy of the nodule is important in establishing diagnosis and initiating therapy.

\section{Conclusion}

To conclude, soft tissue metastasis from a primary lung cancer is unusual and ominous. This case report demonstrates how even a single, new soft tissue lesion can represent a serious occult pathology, thus warranting a low threshold for biopsy and also demonstrates the importance of a good physical examination. The detection of ST metastasis is a poor prognostic indicator, provide more accessible biopsy sites and help avoid invasive procedures, if detected earlier, may be earlier treatment would have offered a better survival probability. 